\title{
El erizo de mar Diadema antillarum Phillipi, 1845 influye sobre la diversidad y composición de la comunidad de mega-invertebrados vágiles en fondos rocosos del Archipiélago Canario
}

\author{
The sea urchin Diadema antillarum Phillipi, 1845 influences the diversity and composition of the \\ mobile mega-invertebrate community on rocky bottoms off the Canary Archipelago \\ Leonor Ortega ${ }^{1}$, Fernando Tuya ${ }^{2,3}$ y Ricardo J. Haroun ${ }^{1}$ \\ ${ }^{1}$ Grupo de Biodiversidad y Gestión Ambiental (BIOGES), Facultad Ciencias Mar, Universidad de Las Palmas de Gran Canaria, \\ 35017, Las Palmas de Gran Canaria, Islas Canarias, España \\ ${ }^{2}$ CIIMAR, Rua dos Bragas 289, 450-123 Porto, Portugal \\ ${ }^{3}$ Center for Ecosystem Management, Edith Cowan University, Joondalup Drive, 6027 Joondalup, WA, Australia \\ leonor129@gmail.com
}

\begin{abstract}
The effect of the sea urchin Diadema antillarum density over the diversity and composition of the mobile megainvertebrate community $(>2 \mathrm{~cm}$ ) inhabiting shallow rocky reefs off the Canary Archipelago (eastern Atlantic) is described. Specifically, it was predicted that (1) high densities of $D$. antillarum produce a low diversity of the mobile megainvertebrate community, and (2) the composition of this community changes between rocky bottoms (= reefs) under different densities of $D$. antillarum. The presence of megainvertebrate taxa, and the density of $D$. antillarum individuals were recorded visually using 2 x 2 m quadrats ( $n=16$ replicates) in each of 24 locations (three locations in each of the eight main islands of the archipelago), which were visited in four occasions between February-2003 and October-2004. Thritythree taxa of mobile mega-invertebrates were observed. We registered a decline in the richness of such assemblages with increasing densities of $D$. antillarum $\left(\mathrm{r}_{\mathrm{s}}=-0.20 ; P<0.001\right)$. Species richness fluctuated considerably (0-16 species) in those locations with low densities of $D$. antillarum $\left(<2\right.$ ind. $\left.\mathrm{m}^{-2}\right)$. However, species richness was always low ( $<9$ species) in those locations with high densities of $D$. antillarum $(>8$ ind. $\mathrm{m}^{-2}$ ). A CAP ordination analysis showed significant differences in the composition of the mega-invertebrate community between rocky bottoms under different densities of $D$. antillarum, and we registered a negative correlation between the presence of some taxa and the densities of sea urchin. Consequently, the sea urchin $D$. antillarum influences the diversity and composition of the mobile mega-invertebrate community on rocky bottoms off the Canary Archipelago.
\end{abstract}

Key words: Echinoderms, community ecology, Canary Islands
Resumen.- Se describe el efecto de la densidad del erizo Diadema antillarum sobre la diversidad y composición de la comunidad de mega-invertebrados vágiles $(>2 \mathrm{~cm})$ que habita fondos rocosos del Archipiélago Canario (Atlántico oriental). Específicamente, se predijo que (1) las altas densidades de $D$. antillarum producen una baja diversidad de la comunidad de mega-invertebrados vágiles, y (2) la composición de las comunidades de mega-invertebrados varía entre fondos con diferentes densidades de $D$. antillarum. La presencia de especies mega-invertebradas, y la densidad de individuos de $D$. antillarum, se registraron visualmente en cuadrados de 2 × $2 \mathrm{~m}$ ( $n=16$ réplicas) en un total de 24 localidades (tres localidades en cada una de las ocho islas principales del archipiélago), visitadas en cuatro ocasiones entre febrero-2003 y octubre-2004. Se observaron 33 taxones de mega-invertebrados vágiles. Se registró una relación inversa entre la riqueza específica y la densidad de $D$. antillarum ( $\left.\mathrm{r}_{\mathrm{s}}=-0,20 ; P<0,001\right)$. En localidades con bajas densidades de $D$. antillarum $\left(<2\right.$ ind. $\left.\mathrm{m}^{-2}\right)$, la riqueza de especies fluctuó considerablemente, entre 0-16 especies. Sin embargo, en localidades con altas densidades de $D$. antillarum ( $>8$ ind. $\mathrm{m}^{-2}$ ), la riqueza de especies fue siempre baja $(<9$ especies). Un análisis de correspondencia canónicas (CAP) mostró diferencias significativas en la composición de la comunidad de mega-invertebrados entre fondos sometidos a diferentes densidades de $D$. antillarum, y se detectó una correlación negativa entre la presencia de ciertas taxa y las densidades de $D$. antillarum. Consecuentemente, el erizo $D$. antillarum influye sobre la diversidad y composición de la comunidad de mega-invertebrados vágiles en fondos rocosos del Archipiélago Canario.

Palabras clave: Equinodermos, ecología de comunidades, Islas Canarias 


\section{Introducción}

Los erizos de mar juegan un papel importante en la diversidad, composición y organización de la biota que habita los fondos rocosos en latitudes templadas (Lawrence 1975, Carpenter et al. 1990, Shears \& Babcock 2003). En general, las altas densidades de estos herbívoros causan cambios cualitativos y cuantitativos en la composición y estructura de las comunidades de organismos sésiles (Knowlton 2004). Usualmente, ocurren disminuciones en la diversidad y abundancia local de comunidades algales (Andrew 1993, Alves et al. 2001, Benedetti-Cecchi et al. 2003, Tuya et al. 2004a,b), y de invertebrados, como consecuencia de la competencia por el substrato (e.g. Pawlik et al. 1995, Edmunds \& Carpenter 2001, Miller et al. 2003, Cebrián \& Uriz 2006). Sin embargo, los efectos que tienen las altas densidades de estos herbívoros sobre la diversidad y estructura de las comunidades de invertebrados bentónicos vágiles, i.e. aquellos con capacidad de movimiento sobre el fondo, podrían mostrar resultados distintos a los encontrados en organismos sésiles. Los invertebrados móviles encuentran alimento y cobijo en los bosques y praderas algales (Begin et al. 2004, Tuya et al. 2008); por tanto, la disminución o desaparición de la cobertura vegetal debido al sobrepastoreo de erizos de mar puede derivar en bajas diversidades y abundancias de especies de invertebrados (Witman \& Dayton 2001).

En el Archipiélago Canario (Atlántico centrooriental), el erizo de púas largas, Diadema antillarum Phillipi, 1845, trasforma áreas cubiertas de algas erectas a substratos carentes de ellas (Tuya et al. 2004a,b). Estas superficies son conocidas localmente como 'blanquizales', debido a su color blanquecino. Son un ejemplo más de fondos deforestados típicos de zonas templadas, como resultado del sobre-pastoreo de los erizos de mar (Lawrence 1975, Sala et al. 1998, Shears $\&$ Babcock 2003, Knowlton 2004). En general, este erizo restringe las macroalgas a los primeros metros del medio submareal, donde la alta turbulencia y la gran disponibilidad lumínica permiten a las algas resistir a su consumidor (Alves et al. 2001, Tuya et al. 2007). El intenso pastaje debido a las altas abundancias de $D$. antillarum produce marcadas transiciones entre las aguas someras vegetadas y áreas desprovistas de vegetación, que se extienden en profundidad (Tuya et al. 2005).

En este estudio, se describe el efecto de la densidad del erizo Diadema antillarum sobre la diversidad y composición de la comunidad de mega-invertebrados vágiles $(>2 \mathrm{~cm})$ habitando fondos rocosos del Archipiélago Canario. Específicamente, se predice que (1) las altas densidades de D. antillarum producen una disminución en la diversidad de la comunidad de megainvertebrados vágiles, y (2) la composición de las comunidades de mega-invertebrados varían entre fondos sometidos a diferentes densidades de D. antillarum.

\section{Material y métodos}

\section{Área de estudio y diseño de muestreo}

Se seleccionaron tres localidades, separadas por decenas de kilómetros, sobre sustratos rocosos en cada una de las ocho islas principales del Archipiélago Canario (Fig. 1). Todas las localidades se seleccionaron con similar pendiente (verticalidad, entre 0 y $15^{\circ}$ de inclinación), para minimizar el efecto del tipo de hábitat sobre los patrones de distribución de las especies (García-Charton \& PérezRuzafa 1999). En todas las localidades muestreadas, la profundidad fluctuó entre 10-18 metros. Cada localidad se visitó en cuatro ocasiones ('campañas') entre febrero2003 y octubre-2004, con una separación aproximada de seis meses entre campañas sucesivas (febrero-marzo 2003; septiembre-octubre 2003; febrero-marzo 2004; septiembre-octubre 2004). La presencia y abundancia de especies mega-invertebradas se registró visualmente, durante la inmersión con equipos autónomos de buceo, mediante la utilización de cuadrados de 2 × 2 m (4 m²), con $n=16$ réplicas por localidad y campaña de muestreo. Los cuadrados se colocaron de manera aleatoria sobre el substrato. Para cada réplica, un buceador procedió a la identificación y anotación en una tablilla provista de papel resistente al agua, de las especies de mega-fauna durante un periodo de tiempo entre 4-5 minutos. La identificación de muchos organismos se ejecutó hasta el nivel de género y/o familia, debido a las limitaciones impuestas por la técnica de muestreo. La densidad de individuos de $D$. antillarum se cuantificó en cada réplica.

Para sucesivos análisis, cada localidad se clasificó en cuatro categorías de acuerdo con la densidad media de individuos de D. antillarum: $<2$ ind. $\mathrm{m}^{-2}, 2-4$ ind. $\mathrm{m}^{-2}$, 4-8 ind. $\mathrm{m}^{-2} \mathrm{y}>8$ ind. $\mathrm{m}^{-2}$. Esta categorización se empleó anteriormente para describir los diferentes estados de organización de los fondos rocosos del Archipiélago Canario (Tuya et al. 2004b).

\section{Análisis de datos}

Un análisis de correlación comprobó la significación de la relación entre la riqueza de especies megainvertebrados y la densidad media de erizos $D$. antillarum por localidad. Se usó la técnica de análisis de correspondencia canónicas (CAP, Anderson \& Willis 2003) para visualizar y contrastar las diferencias en la composición de la comunidad mega-invertebrada entre las cuatro categorías de fondo definidas anteriormente. 


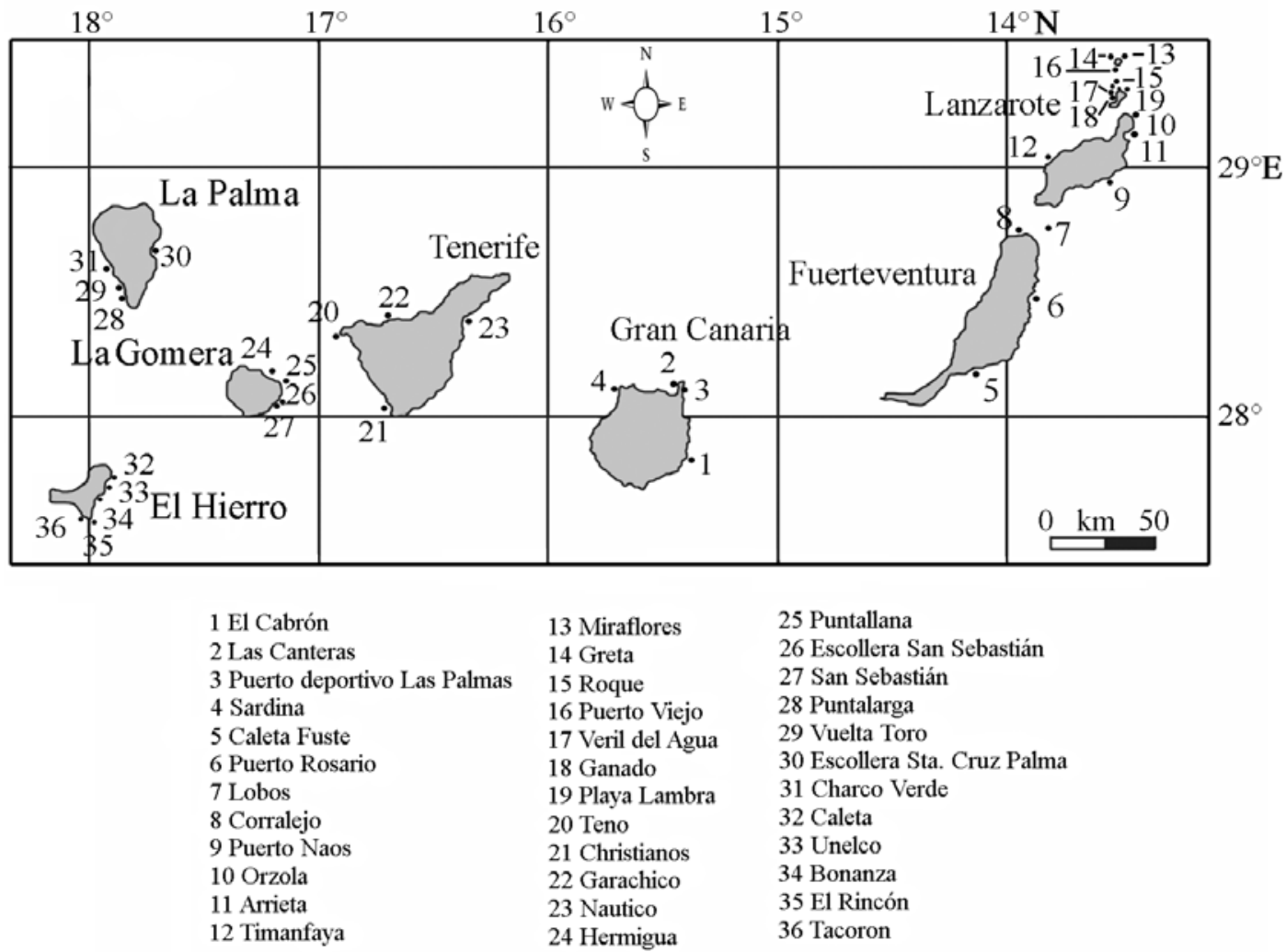

Figura 1

Mapa del Archipiélago Canario mostrando las localidades de muestreo en cada isla

Map of the study area showing the study locations within each island

En esencia, esta técnica busca ejes en el espacio multidimensional que maximicen la separación entre grupos (categorías de fondo en este caso). El análisis se basó en disimilitudes de Jaccard, que son ideales para datos de incidencia (es decir, presencia-ausencia, Clarke \& Warwick 2001). El estadístico 'trace' comprobó, a través de 999 permutaciones de los datos, la significancia de las diferencias en composición de la comunidad de mega-invertebrados entre las cuatro categorías. Finalmente, se empleó el procedimiento no paramétrico ANOSIM para determinar la significancia de las diferencias en la composición de la comunidad megainvertebrada entre cada par de categorías; las interpretaciones del estadístico $\mathrm{R}$ siguieron las indicaciones de Clarke (1993). Todos estos análisis multivariados se ejecutaron con el programa PRIMER 6.0 (Clarke \& Warwick 2001). Para este análisis, se agruparon los datos correspondientes a los cuatro periodos de muestreo ('campañas') para cada localidad.
Un análisis de correlaciones bivariadas (correlación de Spearman) (Clarke \& Warwick 2001) permitió identificar las especies o taxa mega-invertebradas, para las cuales se disponía de datos cuantitativos, que se correlacionaron negativa o positivamente con la densidad de D. antillarum.

\section{Resultados}

Se observaron 33 taxones de mega-invertebrados vágiles a lo largo de la zona de estudio (Tabla 1). Se registró una clara disminución de la riqueza de especies megainvertebradas al incrementarse la densidad media de erizos por localidad $\left(r_{s}=-0,20, P<0,001\right.$, Fig. 2$)$. En localidades con bajas densidades de erizos $\left(<2\right.$ ind. $\left.\mathrm{m}^{-2}\right)$, la riqueza de especies fluctuó considerablemente (entre 0-16 especies, Fig. 2). Sin embargo, la riqueza de especies fue siempre baja y, en todo los casos, inferior a 9 especies (Fig. 2), en localidades con altas densidades de $D$. antillarum $\left(>8\right.$ ind. $\left.\mathrm{m}^{-2}\right)$. 


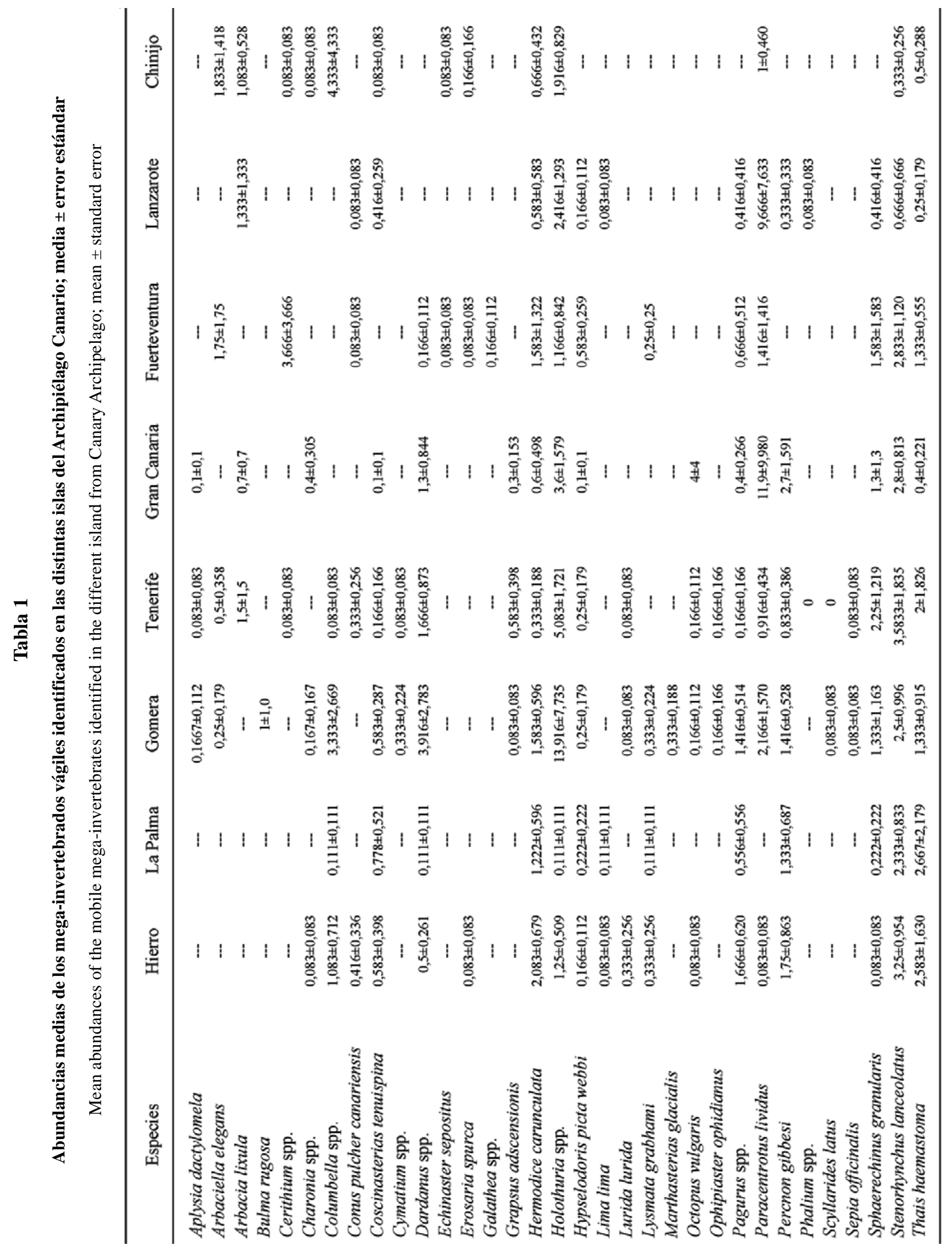




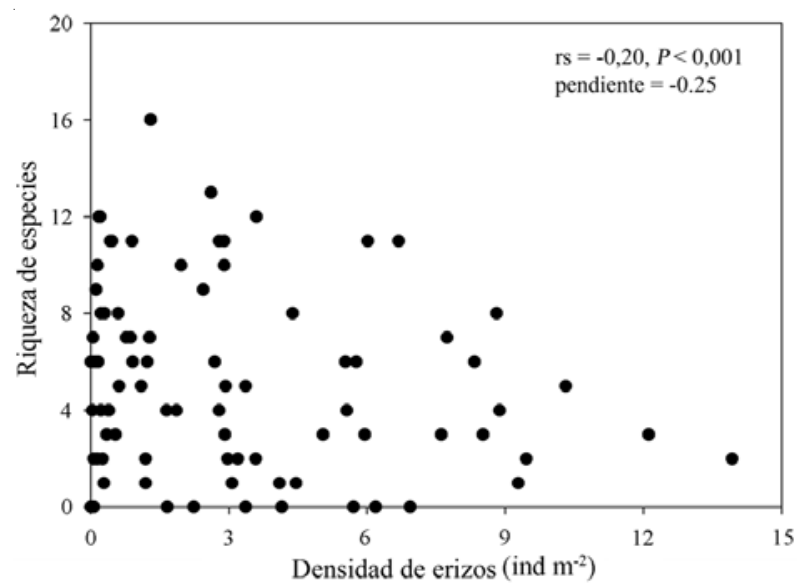

Figura 2

Relación entre la riqueza de especies de megainvertebrados vágiles y la densidad media de erizos $D$. antillarum por localidad

Relationship between richness of mobile mega-invertebrates and mean density of sea urchin D. antillarum by location

El análisis de ordenación CAP separó eficientemente las comunidades mega-invertebradas correspondientes a las cuatro categorías de fondo consideradas a lo largo de primer eje canónico (Fig. $3, \delta_{1}^{2} P<0,01$ ); por ejemplo, las localidades con bajas densidades de erizos tendieron a agruparse en la parte inferior derecha del diagrama. Sin embargo, estas diferencias en la composición de las comunidades mega-invertebradas entre categorías de fondo solamente fueron significativas entre fondos con densidades de $D$. antillarum entre $0-2$ ind. $\mathrm{m}^{-2} \mathrm{y}$ fondos con densidades $>8$ ind. $\mathrm{m}^{-2}$ (ANOSIM, R-estadístico $=$ $0,14 ; P=0,04)$.

El análisis de correlaciones mostró que la abundancia de cangrejos los ermitaños Pagurus spp., Dardanus spp., el erizo Spharachinus granularis, y el molusco Aplysia dactylomela se correlacionaron $(P<0,05)$ negativamente con la abundancia de D. antillarum. Únicamente el crustáceo decápodo Stenorhynchus lanceolatus presentó una correlación positiva $(P<0,05)$ con la abundancia de D. antillarum.

\section{Discusión}

Este estudio ha mostrado el papel relevante que juega el erizo negro de púas largas, Diadema antillarum, como agente determinante de la diversidad y composición de

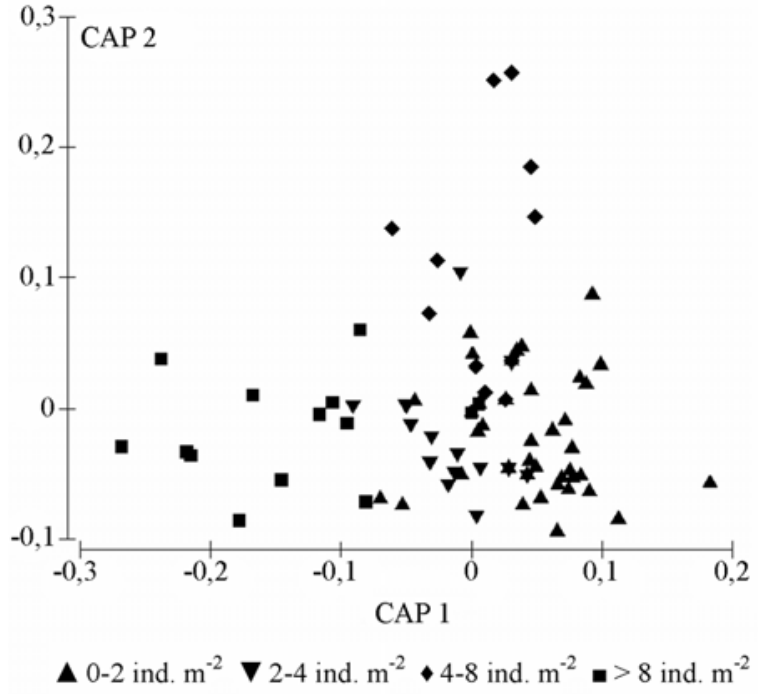

Figura 3

Diagrama de ordenación CAP mostrando los cambios en composición de la comunidad de mega-invertebrados sobre fondos rocosos del Archipiélago Canario en función de la densidad de $D$. antillarum

Analysis of ordination CAP showing changes in composition of the mega invertebrate community on rocky bottoms from Canary Archipelago in relation to density of $D$. antillarum

comunidades mega-invertebradas sobre fondos rocosos someros del Archipiélago Canario. En general, los incrementos en densidad de este erizo de mar se han correlacionado con una disminución en la riqueza de especies mega-invertebradas, culminando en un cambio claro en la composición de las comunidades megainvertebradas entre fondos con elevadas $\left(>8\right.$ ind. $\left.\mathrm{m}^{-2}\right) \mathrm{y}$ bajas $\left(<2\right.$ ind. $\mathrm{m}^{-2}$ ) densidades de $D$. antillarum. Este resultado corrobora las observaciones realizadas sobre arrecifes artificiales en el Archipiélago Canario, donde el erizo D. antillarum determinó los patrones de sucesión ecológica sobre los mismos (Herrera 1998); el reclutamiento de individuos de $D$. antillarum produjo una caída en la diversidad algal, así como en la diversidad de la comunidad de macro-invertebrados.

En el Archipiélago Canario, D. antillarum es el principal herbívoro sobre fondos rocosos; cuando las densidades alcanzan los 10 ind. $\mathrm{m}^{-2}$, este erizo puede llegar a consumir la totalidad de la producción primaria bentónica (Tuya et al. 2004b). La desaparición de la biomasa algal supone la pérdida del hábitat para numerosas especies de peces (Tuya et al. 2005), limitando 
así zonas potenciales de reproducción, cría y alimentación. De igual forma, la disminución en la diversidad (riqueza) de especies mega-invertebradas detectadas por este estudio en fondos con altas densidades del erizo $D$. antillarum podría deberse tanto a la escasez de alimento, como a una disminución en la disponibilidad de refugio, o a una combinación de ambas. Los bosques y praderas algales juegan un papel importante como elemento reductor de las tasas de depredación que ejercen depredadores, como los peces, sobre invertebrados bentónicos (Roberts \& Poore 2005), además de reducir los niveles de estrés ambiental, por ejemplo el inducido por fuerzas hidrodinámicas (Bruno et al. 2003), y proporcionar lugares de puesta a numerosos invertebrados bentónicos.

Los cambios en la organización de los sistemas bentónicos no afectan por igual a todas las especies; la presencia de estados alternativos para un mismo sistema (e.g. 'forestado’ vs. ‘deforestado’) producen cambios en la composición de especies, y unas especies son reemplazadas por otras (Shears \& Babcock 2003, Knowlton 2004). Por ejemplo, la abundancia de algunas especies de holoturias puede ser favorecida localmente por las altas densidades del erizo D. antillarum en el Archipiélago Canario (Tuya et al. 2006). En nuestro estudio, sin embargo, no se detectó dicha correlación, debido probablemente a que el cambio en la escala espacial de los muestreos condiciona dicha relación. Por otro lado, las altas densidades de erizos de púas largas (D. antillarum) facilitan altas densidades del crustáceo decápodo Stenorinchus lanceolatus. Este patrón está vinculado, probablemente, a que este cangrejo encuentra refugio entre las largas púas de los erizos, lo que ofrece un refugio ante sus depredadores (Espino et al. 2006). También, se detectó que ciertas taxa se correlacionaron negativamente con las densidades del erizo $D$. antillarum a lo largo de todo el Archipiélago. Por ejemplo, los cangrejos ermitaños Pagurus spp. y Dardanus spp. o el molusco Aplysia dactylomela encuentran refugio y alimento en fondos rocosos vegetados del Archipiélago Canario (Espino et al. 2006). La desaparición de dicha vegetación posiblemente les afecta negativamente, tal y como se comentó anteriormente.

En conclusión, nuestro estudio ha demostrado que el erizo Diadema antillarum influye sobre la diversidad y composición de la comunidad de mega-invertebrados vágiles en fondos rocosos del Archipiélago Canario.

\section{Agradecimientos}

Este estudio se realizó gracias a la financiación del Ministerio de Medio Ambiente del Gobierno de España en el marco del proyecto Canarias, Costa Viva. Dos revisores anónimos proporcionaron comentarios que ayudaron a mejorar una versión anterior del manuscrito. Agradecemos a A. Boyra, P. Sánchez-Jerez, C. Barberá, O. Bergasa, T. Sánchez, A. Iglesias, A. López, N. Rodriguez, A. Del Rosario, G. Herrera, R. Herrera y F. Espino su ayuda durante el trabajo de campo.

\section{Literatura citada}

Alves FMA, LM Chicharo, E Serrao \& AD Abreu. 2001. Algal cover and sea-urchin spatial distribution at Madeira Island (NE Atlantic). Scientia Marina 65: 383-392.

Anderson MJ \& TJ Willis. 2003. Canonical analysis of principal coordinates: a useful method of constrained ordination for ecology. Ecology 84: 511-525.

Andrew NL. 1993. Spatial heterogeneity, sea urchin grazing, and habitat structure on reefs in temperate Australia. Ecology 74: 292-302.

Begin C, LD Johnson \& JH Himmelman. 2004. Macroalgal canopies: distribution and diversity of associated invertebrates and effects on the recruitment and growth of mussels. Marine Ecology Progress Series 271: 121-132.

Benedetti-Cecchi L, F Bulleri \& F Cinelli. 1998. Density dependent foraging of sea urchins in shallow subtidal reefs on the west coast of Italy (western Mediterranean). Marine Ecology Progress Series 163: 203-211.

Bruno JF, JJ Stachowicz \& MD Bertness. 2003. Inclusion of facilitation into ecological theory. Trends in Ecology \& Evolution 18: 119-125.

Carpenter RC. 1990. Mass mortality of Diadema antillarum. II. Effects on population densities and grazing intensity of parrotfishes and surgeonfishes. Marine Biology 104: 7986.

Cebrián E \& MJ Uriz. 2006. Grazing on fleshy seaweeds by sea urchins facilitates sponge Cliona viridis growth. Marine Ecology Progress Series 323: 83-89.

Clarke KR. 1993. Non-parametric multivariate analyses of changes in community structure. Australian Journal of Ecology 18: 117-143.

Clarke KR \& RM Warwick. 2001. Changes in marine communities: an approach to statistical analysis and interpretation, 144 pp. PRIMER-E, Plymouth.

Edmunds PJ \& RC Carpenter. 2001. Recovery of Diadema antillarum reduces macroalgal cover and increases abundance of juvenile corals on a Caribbean reef. Proceedings of the National Academy of Sciences of the United States of America 98: 5067-5071.

Espino F, A Boyra, F Tuya \& RJ Haroun. 2006. Guía visual de las especies marinas de Canarias, 482 pp. Ediciones Oceanográficas, Telde.

García-Charton JA \& A Pérez-Ruzafa. 1999. Ecological heterogeneity and the evaluation of the effects of marine reserves. Fisheries Research 42: 1-20. 
Herrera R. 1998. Dinámica de las comunidades bentónicas de los arrecifes artificiales de Arguineguín (Gran Canaria) y Lanzarote. Tesis Doctoral, Universidad de Las Palmas de Gran Canaria, Las Palmas de Gran Canaria, 355 pp.

Knowlton N. 2004. Multiple 'stable' states and the conservation of marine ecosystems. Progress in Oceanography 60: 387396.

Lawrence JM. 1975. On the relationships between marine plants and sea urchins. Oceanography and Marine Biology: an Annual Review 13: 213-286.

Miller RJ, AJ Adams, NB Ogden, JC Ogden \& JP Ebersole. 2003. Diadema antillarum 17 years after mass mortality: is recovery beginning on St. Croix? Coral Reefs 22: 181187.

Pawlik JR, B Chanas, RJ Toonen \& W Fenical. 1995. Defenses of Caribbean sponges against predatory reef fish: I. Chemical deterrency. Marine Ecology Progress Series 127: 183-194.

Roberts DA \& AGB Poore. 2005. Habitat configuration affects colonization of epifauna in a marine algal bed. Biological Conservation 127: 18-26.

Sala E, CF Boudouresque \& ML Harmelin-Vivien. 1998. Fishing, trophic cascades, and the structure of algal assemblages: evaluation of an old but untested paradigm. Oikos 83: 425-439.

Shears NT \& RC Babcock. 2003. Continuing trophic cascade effects after 25 years of no-take marine reserve protection. Marine Ecology Progress Series 246: 1-16.
Tuya F, JA Martín \& A Luque. 2004a. Patterns of nocturnal movement of the long-spined sea urchin Diadema antillarum (Philippi) in Gran Canaria (the Canary Islands, central East Atlantic Ocean). Helgoland Marine Research 58: 26-31.

Tuya F, A Boyra, P Sánchez-Jerez, C Barberá \& RJ Haroun. 2004b. Relationships between rocky-reef fish assemblages, the sea urchin Diadema antillarum and macroalgae throughout the Canarian Archipelago. Marine Ecology Progress Series 278: 157-169.

Tuya F, RJ Haroun, A Boyra \& P Sánchez-Jerez. 2005. Sea urchin Diadema antillarum: different functions in the structure and dynamics of reefs on both sides of the Atlantic. Marine Ecology Progress Series 302: 307-310.

Tuya F, JC Hernández \& S Clemente. 2006. Is there a link between the type of habitat and the patterns of abundance of holothurians in shallow rocky reefs? Hydrobiologia 571: 191-199.

Tuya F, J Cisneros-Aguirre, L Ortega-Borges \& RJ Haroun. 2007. Bathymetric segregation of sea urchins on reefs of the Canarian Archipelago: role of flow-induced forces. Estuarine, Coastal and Shelf Science 73: 481-488.

Tuya F, T Wernberg \& MS Thomsen. 2008. The spatial arrangement of reefs alters the ecological patterns of fauna between interspersed habitats. Estuarine, Coastal and Shelf Science 78: 774-782.

Witman JD \& P Dayton. 2001. Rocky subtidal communities. En: Bertness MD, SD Gaines \& ME Hay (eds). Marine Community ecology, pp. 339-366. Sinauer Associates, Sunderland.

Recibido el 29 de enero de 2009 y aceptado el 10 de junio de 2009 